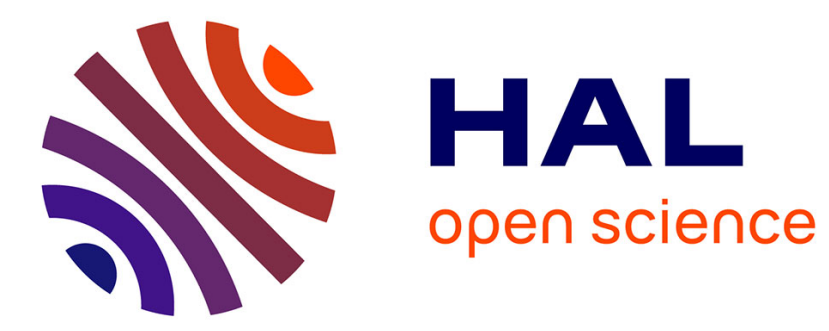

\title{
Premières dates radiocarbone pour le Magdalénien d'Isturitz (Pyrénées-Atlantiques)
}

Carolyn Szmidt, Jean-Marc Pétillon, Pierre Cattelain, Christian Normand, Catherine Schwab

\section{- To cite this version:}

Carolyn Szmidt, Jean-Marc Pétillon, Pierre Cattelain, Christian Normand, Catherine Schwab. Premières dates radiocarbone pour le Magdalénien d'Isturitz (Pyrénées-Atlantiques). Bulletin de la Société préhistorique française, 2009, 106 (3), pp.588-592. halshs-00461782

\section{HAL Id: halshs-00461782 \\ https://shs.hal.science/halshs-00461782}

Submitted on 1 Jul 2012

HAL is a multi-disciplinary open access archive for the deposit and dissemination of scientific research documents, whether they are published or not. The documents may come from teaching and research institutions in France or abroad, or from public or private research centers.
L'archive ouverte pluridisciplinaire HAL, est destinée au dépôt et à la diffusion de documents scientifiques de niveau recherche, publiés ou non, émanant des établissements d'enseignement et de recherche français ou étrangers, des laboratoires publics ou privés. 
Premières dates radiocarbone pour le Magdalénien d'Isturitz (Pyrénées-Atlantiques)

Situé au carrefour de la plaine aquitaine, des Pyrénées, de la haute vallée de l'Èbre et de la côte cantabrique, le site d'Isturitz a joué un rôle pivot dans l'occupation du Sud-Ouest européen pendant une grande partie du Paléolithique supérieur, et plus particulièrement lors de la période magdalénienne. Durant les phases moyenne et supérieure de cette culture, la cavité a en effet été intensément occupée, comme en témoignent les abondantes collections issues des fouilles Passemard et Saint-Périer, conservées pour l'essentiel au musée d'Archéologie nationale. Certaines matières premières lithiques attestent de relations avec le nord (Bassin aquitain) et le sud (haute vallée de l'Èbre : Normand, obs. pers.; Langlais, 2007). De nombreux types d'objets en matière osseuse se retrouvent aussi bien à l'est dans les Pyrénées qu'à l'ouest sur la côte cantabrique : c'est le cas par exemple des pointes à base fourchue (Pétillon, 2006) ou encore des contours découpés (synthèse dans Cattelain et Bellier, sous presse). La présence d'armatures en os de cétacé (Pétillon, 2008) montre également des relations avec la zone littorale, alors située à 50-60 km. Au Magdalénien moyen et supérieur, la grotte d'Isturitz se trouve donc au cœur d'un réseau de circulation s'étendant dans toutes les directions jusqu'à plusieurs centaines de kilomètres. Elle fait partie des gisements majeurs du Magdalénien franco-cantabrique, dont elle a d'ailleurs contribué à définir les caractéristiques chronoculturelles, mais paradoxalement sans avoir fait jusqu'ici l'objet de datations radiométriques.

Nous avons lancé un programme de datations radiocarbone par spectrométrie de masse par accélérateur (SMA) afin de préciser la chronologie du Magdalénien d'Isturitz, dans le cadre d'une recherche plus générale sur les évolutions internes de cette culture. Nous présentons dans cette note les méthodes, les premiers résultats et le contexte dans lequel ils s'inscrivent. Ce programme a été financé par une bourse de la fondation des Treilles, fondation que nous tenons à remercier ici.

\section{Stratigraphie du Magdalénien d'Isturitz et problématique chronologique}

La grotte d'Isturitz est divisée en deux salles principales : la salle de Saint-Martin (ou salle sud) et la Grande Salle (ou salle d'Isturitz, ou salle nord). Dans la salle de Saint-Martin, les fouilleurs successifs - E. Passemard puis R. et S. de Saint-Périer - mentionnent une seule couche magdalénienne, E $\omega$ pour Passemard et SI pour les Saint-Périer, attribuée au Magdalénien moyen. Dans la Grande Salle, ils en distinguent deux, respectivement rattachées au Magdalénien moyen et supérieur : E et F1 pour Passemard, II et I pour les Saint-Périer.

Une révision récente de cette stratigraphie (Pétillon, 2004) a permis de confirmer globalement l'attribution des différentes couches, ainsi que l'équivalence entre les nomenclatures des deux fouilleurs. Nous parlerons donc des couches SI/Ew (Magdalénien moyen de la salle de SaintMartin), II/E (Magdalénien moyen de la Grande Salle) et I/F1 (Magdalénien supérieur de la Grande Salle).
Ces travaux récents ont cependant soulevé plusieurs interrogations concernant la chronologie du Magdalénien d'Isturitz. La couche II/E se singularise ainsi par la présence d'éléments généralement attribués au début du Magdalénien moyen - telles les pointes à biseau simple de type Lussac-Angles - associés à des éléments renvoyant plutôt au Magdalénien moyen «classique» des Pyrénées (baguettes demi-rondes à décor de tubercules ou de volutes, rondelles percées, contours découpés, propulseurs...). Certaines indications tirées des publications de Passemard et Saint-Périer appuient par ailleurs l'hypothèse de subdivisions internes de cette couche (Pétillon, 2004, p. 127-129). A contrario, la couche SI/E $\omega$ a livré une industrie osseuse plus homogène, marquée notamment par l'absence de pointes à biseau simple; ce caractère est confirmé par le matériel magdalénien issu des fouilles actuelles de la salle de Saint-Martin (Normand dir., 2008). Cette homogénéité pouvait suggérer que le Magdalénien moyen de cette salle correspondait à une phase d'occupation plus courte que dans la salle voisine (Pétillon, ibid.). Par ailleurs, dans la couche I/F1, des indices - relative rareté et caractère «archaïque» des pointes barbelées, forte proportion de pointes à base fourchue parmi les armatures osseuses - suggéraient pour le Magdalénien supérieur d'Isturitz une date précoce, immédiatement postérieure aux ultimes manifestations du Magdalénien moyen, voire contemporaines de celles-ci (Pétillon, 2006, p. 211-218).

\section{Sélection des artefacts}

Les artefacts analysés (tabl. 1 et fig. 1) ont été choisis afin de tester ces hypothèses chronologiques. L'ancienneté des fouilles Passemard et Saint-Périer (1912-1937 pour les couches magdaléniennes) rendait hasardeuse la datation de vestiges fauniques. Les pièces ont donc été choisies parmi les vestiges d'industrie osseuse typologiquement caractéristiques; elles ont été sélectionnées collectivement par les auteurs de cet article, parmi les collections conservées au musée d'Archéologie nationale, en tenant compte de paramètres de différentes natures (protocole technique de datation, critères archéologiques et muséologiques). Ces objets ont été étudiés et photographiés avant analyse.

C'est grâce à la méthode par SMA, qui ne nécessite qu'une quantité de matière très réduite, que ce type d'analyse a pu être entrepris sans trop endommager les vestiges. L'usage de la méthode «classique» aurait entraîné soit la datation de lots de vestiges fauniques dont la provenance stratigraphique n'était pas forcément homogène, soit la destruction d'un lot important de vestiges d'industrie osseuse - deux options peu satisfaisantes.

Trois pièces ont été choisies pour chaque couche. Pour la couche SI/E $\omega$, nous avons retenu un fragment mésial de baguette demi-ronde présentant une rainure longitudinale sur la face supérieure, ainsi qu'un fragment mésial de pointe montrant sur chaque bord une rainure longitudinale dont le fond porte des incisions obliques. Ces deux pièces, rattachées à l'équipement de chasse (fragments d'armature), sont particulièrement fréquentes dans l'industrie osseuse de cette couche. Le troisième objet est un fragment de scapula gauche de Renne portant des traces techniques compatibles avec l'extraction d'une rondelle. 


\begin{tabular}{|c|c|c|c|c|c|c|c|c|c|c|c|c|c|c|c|}
\hline 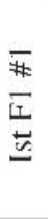 & 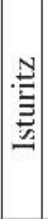 & $\vec{\Xi}$ & 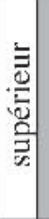 & 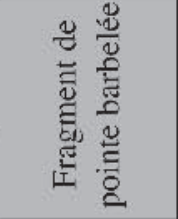 & 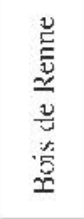 & 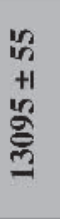 & $\begin{array}{l}\frac{2}{n} \\
\frac{1}{n} \\
\stackrel{n}{n} \\
\infty \\
n \\
n\end{array}$ & छे & $\begin{array}{l}5 \\
7\end{array}$ & $r$ & ซ் & $\begin{array}{l}m \\
2 \\
2 \\
1\end{array}$ & $m$ & ${ }_{n}$ & $\left|\begin{array}{l}3 \\
2 \\
0 \\
2 \\
\hdashline \\
\vdots \\
6 \\
0\end{array}\right|$ \\
\hline 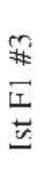 & 尝 & $\overrightarrow{5}$. & 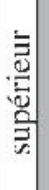 & 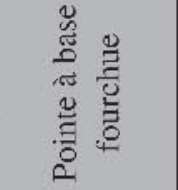 & 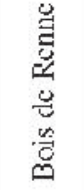 & 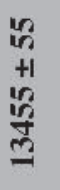 & $\begin{array}{l}\overline{3} \\
0 \\
n \\
1 \\
\vdots \\
0 \\
0\end{array}$ & छ్ & $\begin{array}{l}2 \\
8 \\
\Omega\end{array}$ & $\vec{n}$ & गे & $\frac{n}{2}$ & $\begin{array}{l}\infty \\
\text { in }\end{array}$ & ${ }^{+}$ & $\left|\begin{array}{l}n \\
2 \\
0 \\
0 \\
0 \\
2 \\
5 \\
0 \\
0\end{array}\right|$ \\
\hline$\frac{\mathbb{N}}{\mathbb{W}}$ & 总 & $\overline{5}$. & 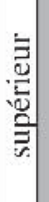 & 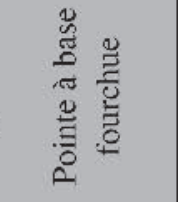 & 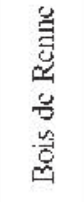 & 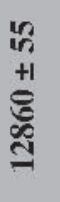 & 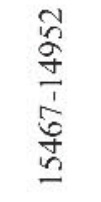 & 8 & $\stackrel{0}{=}$ & $N$ & $\begin{array}{l}a \\
\infty \\
m\end{array}$ & 焉 & + & m. & $\begin{array}{c} \pm \\
0 \\
0 \\
2 \\
\vdots \\
\vdots \\
0 \\
0\end{array}$ \\
\hline 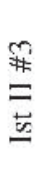 & 莺 & 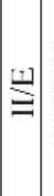 & 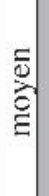 & 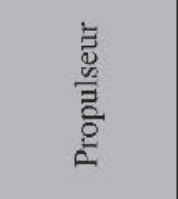 & 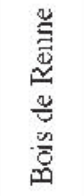 & 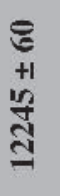 & $\begin{array}{l}\frac{5}{2} \\
\frac{2}{1} \\
\frac{1}{8} \\
\frac{9}{2}\end{array}$ & $\S$ & $\infty$ & $=$ & ְุ. & की & in & $m$ & $\left|\begin{array}{l}5 \\
0 \\
\infty \\
2 \\
0 \\
2 \\
5 \\
0\end{array}\right|$ \\
\hline 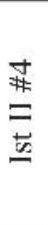 & 总 & $\stackrel{1}{=}$ & 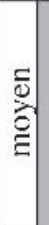 & 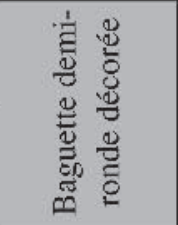 & 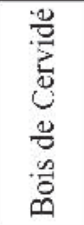 & 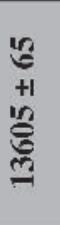 & 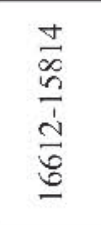 & $\S$ & $\infty$ & ले & $\frac{\nabla}{8}$ & $\frac{m}{2}$ & $-\bar{m}$ & 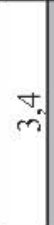 & 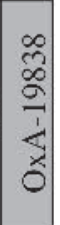 \\
\hline $\begin{array}{l}\overline{\#} \\
\bar{E} \\
\underline{E}\end{array}$ & 羙 & $\stackrel{\Xi}{=}$ & 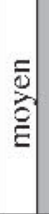 & 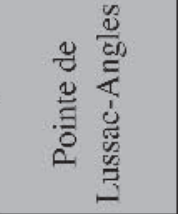 & 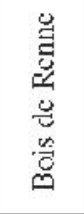 & 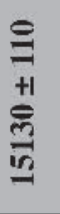 & $\begin{array}{l}\stackrel{\infty}{\widehat{\infty}} \\
\frac{1}{\tilde{N}} \\
\stackrel{\infty}{\infty}\end{array}$ & $\S$ & $=$ & $\vec{f}$ & $\stackrel{\mathfrak{f}}{\mathfrak{f}}$ & \begin{tabular}{c}
0 \\
2 \\
0 \\
\hdashline \\
1
\end{tabular} & il & लै & $\begin{array}{c}0 \\
\infty \\
2 \\
2 \\
\\
\vdots \\
0\end{array}$ \\
\hline $\begin{array}{l}\bar{W} \\
\bar{\sigma} \\
\underline{\underline{a}}\end{array}$ & 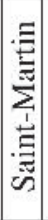 & :3) & 를 & 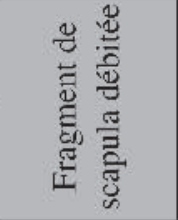 & 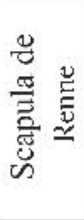 & 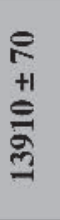 & 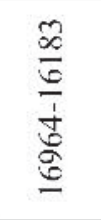 & 워 & $\Rightarrow$ & $\Rightarrow$ & $\begin{array}{l}9 \\
\text { जे }\end{array}$ & ज̂ & $\sigma_{0}$ & 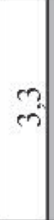 & 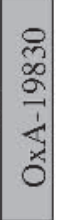 \\
\hline 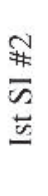 & 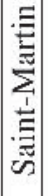 & 羕 & है & 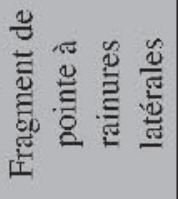 & 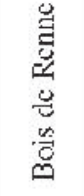 & $\begin{array}{l}\bar{\Xi} \\
+1 \\
\stackrel{+}{\Xi} \\
\exists \\
\exists\end{array}$ & $\begin{array}{l}\frac{N}{3} \\
\frac{\sigma}{1} \\
\frac{\sigma}{2}\end{array}$ & 잉 & $\begin{array}{l}3 \\
\mathrm{n} \\
\mathrm{n}\end{array}$ & gे & $\vec{f}$ & $\left|\begin{array}{l}\bar{\infty} \\
\infty \\
-\infty\end{array}\right|$ & 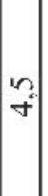 & $\mathrm{m}$ & $\begin{array}{l}3 \\
0 \\
2 \\
\\
\vdots \\
\vdots \\
0\end{array} \mid$ \\
\hline 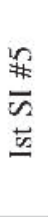 & 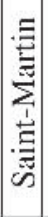 & 离 & 矛 & 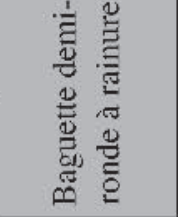 & 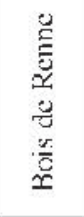 & 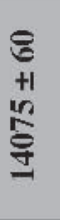 & 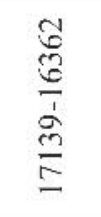 & ถิ & 放 & ? & $\stackrel{n}{+}$ & 包 & $\nabla$ & 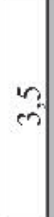 & $\begin{array}{l}0 \\
2 \\
2 \\
2 \\
\vdots \\
\vdots \\
0 \\
0\end{array}$ \\
\hline 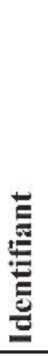 & 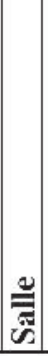 & : & 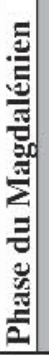 & 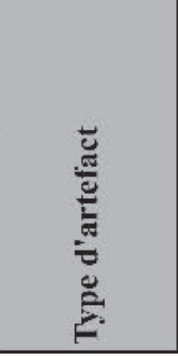 & 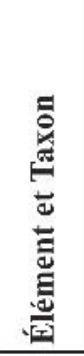 & 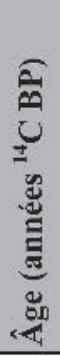 & 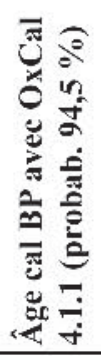 & 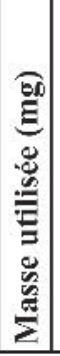 & 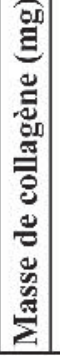 & 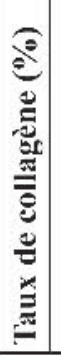 & U & 章 & 章 & 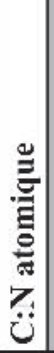 & 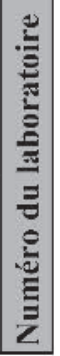 \\
\hline
\end{tabular}

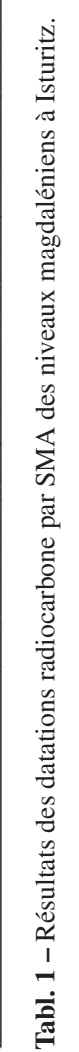


De la couche II/E, nous avons sélectionné une pointe à biseau simple de type Lussac-Angles, un fragment de baguette demi-ronde orné de tubercules et une ébauche de propulseur. Cette dernière pièce a été choisie car le site d'Isturitz a livré plus de dix parties distales de propulseur certaines ou probables, typologiquement variées, provenant des couches du Magdalénien moyen mais dont le cadre chronologique demandait à être précisé (Cattelain, sous presse).

Enfin, la couche I/F1 est représentée par deux fragments de pointes à base fourchue - type d'armature osseuse largement dominant dans cette couche (Pétillon, 2006) - et un fragment mésial de pointe à une rangée de barbelures $(\ll$ harpon $»)$.

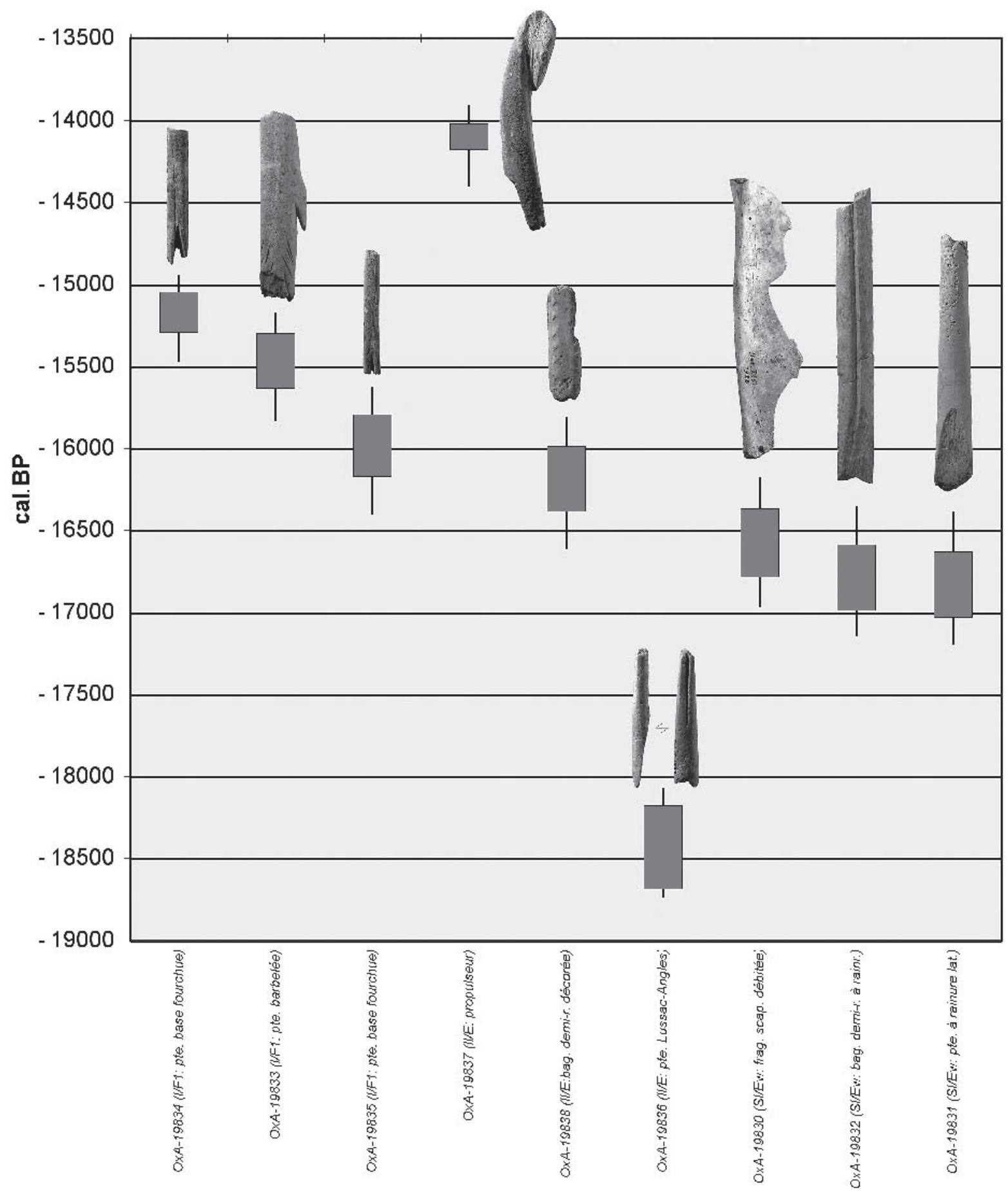

Fig. 1 - Dates calibrées (avec OxCal 4.1.1) des niveaux magdaléniens à Isturitz. Les dates sont présentées par couche, en ordre croissant d'âge. Les trois échantillons provenant des couches du Magdalénien supérieur de la salle d'Isturitz (I/F1) sont à gauche dans le graphe. Ceux du Magdalénien moyen de cette salle (II/E) se trouvent au centre et les trois de la salle de Saint-Martin (SI/E $\omega)$ sont à droite. Le rectangle représente la tranche de dates calibrées de probabilité $68,2 \%$; les traits représentent la tranche de dates calibrées de probabilité $94,5 \%$. Il est à noter que pour l'échantillon OxA-19836, de multiples tranches de dates ont résulté du programme de calibration : 18688-18472 cal. BP (probabilité 45,5\%); 18336-18176 cal. BP (probabilité 22,7\%). Celles-ci ont été combinées dans ce graphe. La tranche de probabilité 94,5 \% pour cet échantillon est de 18732-18076 cal. BP. 


\section{Méthodologie et résultats}

Les artefacts ont été analysés au Oxford Radiocarbon Accelerator Unit (ORAU), laboratoire de datation de l'université d'Oxford. Ils ont été traités par la méthode dite de Longin (1971) d'extraction du collagène, avec l'étape d'ultrafiltration actuellement utilisée par ce laboratoire (Bronk Ramsey et al., 2004a; Brock et al., 2007). La combustion, la graphitisation et la mesure ont été effectuées selon les procédures établies par le laboratoire (Bronk Ramsey et al., 2002 et 2004b). Les artefacts n'avaient été recouverts ni d'agents de conservation ni de consolidant. De plus, aucun moulage n'a été fait de ces pièces.

Les âges radiocarbone non calibrés exprimés en années radiocarbone BP (Before Present : avant 1950) ont été calculés en utilisant la valeur Libby de la demi-vie du ${ }^{14} \mathrm{C}$ et corrigés du fractionnement isotopique naturel. Le taux de collagène, ainsi que les mesures élémentaires et d'isotopes stables entreprises sur les échantillons (C:N, $\left.\delta^{13} \mathrm{C}, \delta^{15} \mathrm{~N}\right)$ se situent toutes dans des marges normales (tabl. 1) (Burleigh et al., 1984; DeNiro, 1985; Hedges et Van Klinken, 1992; Van Klinken, 1999). La calibration des dates (tabl. 1 et fig. 1) a été effectuée avec le programme OxCal (v. 4.1.1., 2009), développé par C. Bronk Ramsey (2001 et sous presse), en se basant sur les données de IntCal04.

\section{Discussion}

Les trois dates obtenues pour la couche SI/Ew sont groupées autour de 16500-17000 cal. BP, ce qui est cohérent avec l'homogénéité constatée dans la composition de l'industrie osseuse et correspond chronologiquement au Magdalénien moyen «classique» des Pyrénées. Deux des trois dates de la couche II/E soulignent au contraire l'hétérogénéité du Magdalénien moyen de la Grande Salle: si les pointes de Lussac-Angles semblent bien relever d'une phase très ancienne du Magdalénien moyen, la date obtenue sur la baguette demi-ronde décorée nous place au contraire à la fin de cette même période. Quant aux dates de la couche I/F1, elles confirment la présence à Isturitz d'un Magdalénien "supérieur ancien", livrant des pointes à base fourchue et des pointes barbelées, à des dates situées pendant la transition entre l'événement de Heinrich 1 et le réchauffement du GIS-1e (ou Bølling).

Reste la date de 13917-14401 cal. BP (probabilité 94,5\%), obtenue sur l'ébauche de propulseur, qui ne cadre pas avec l'attribution de la couche II/E au Magdalénien moyen. Aucun argument ne permet cependant de mettre en doute la fiabilité de cette mesure par rapport aux huit autres. Par ailleurs, l'ancienneté des fouilles SaintPérier ne permet pas d'exclure la présence, dans cette couche, de pièces intrusives en provenance des niveaux plus récents (Pétillon, 2004). Si on retient cette hypothèse, on en vient donc à envisager d'attribuer l'ébauche de propulseur au Magdalénien supérieur. Reste que la date de cette pièce est plus récente que les trois dates du Magdalénien supérieur de la couche I/F1 sus-jacente... La date obtenue sur le propulseur signalerait-elle la présence à Isturitz d'un Magdalénien «supérieur récent», se plaçant pendant le Bølling, et dont ne proviendrait aucune des trois pièces datées dans la couche $\mathrm{I} / \mathrm{F} 1$ ? Cette hypothèse reste à discuter.
Quoi qu'il en soit, ces résultats confortent la place d'Isturitz comme l'une des séquences de référence pour la chronologie du Magdalénien franco-cantabrique. Ces premières données radiométriques devront certes être complétées; mais l'objectif de cette note est de faire en sorte qu'elles viennent d'ores et déjà alimenter la discussion sur la chronologie du Magdalénien moyen et supérieur à l'échelle du Sud-Ouest européen.

\section{RÉFÉRENCES BIBLIOGRAPHIQUES}

BROCK F., BRONK RAMSEY C., HIGHAM T. (2007) - Quality assurance of ultrafiltered bone dating, Radiocarbon, vol. 49, $\mathrm{n}^{\circ} 2$, p. 187 192.

BRONK RAMSEY C. (2001) - Development of the Radiocarbon calibration Program OxCal, Radiocarbon, vol. 43, p. 355-363.

BRONK RAMSEY C. (sous presse) - Bayesian analysis of radiocarbon dates, Radiocarbon.

BRONK RAMSEY C., HIGHAM T.F.G., OWEN D.C., PIKE A.W.G., HEDGES R.E.M. (2002) - Radiocarbon dates from the Oxford AMS system: Archaeometry datelist 31, Archaeometry, vol. 44, n 3 , suppl. 1, p. 1-149.

BRONK RAMSEY C., HIGHAM T., BOWLES A., HEDGES R. (2004a) - Improvements to the pretreatment of bone at Oxford, Radiocarbon, vol. $46, \mathrm{n}^{\circ} 1$, p. 155-163.

BRONK RAMSEY C., HIGHAM T., LEACH P. (2004b) - Towards high-precision AMS: Progress and limitations, Radiocarbon, vol. 46, $\mathrm{n}^{\circ} 1$, p. 17-24.

BURLEIGH R., MATTHEWS K., LEESE M. (1984) - Consensus $\delta^{13} \mathrm{C}$ values, Radiocarbon, vol. $26, \mathrm{n}^{\circ} 1$, p. 46-53.

CATTELAIN P. (sous presse) - Les propulseurs d'Isturitz, in C. Normand dir., Les recherches archéologiques dans les grottes d'Isturitz et d'Oxocelhaya de 1912 à nos jours : une synthèse des résultats.

CATTELAIN P., BELLIER C. (sous presse) - Objets décorés pris sur stylohyoideum, Industrie de l'os préhistorique, cahier XIII, Matières d'art.

DeNIRO M.J. (1985) - Postmortem preservation and alteration of in vivo bone collagen isotope ratios in relation to palaeodietary reconstruction, Nature, vol. 317, p. 806-809.

HEDGES R.E.M., VAN KLINKEN G.J. (1992) - A review of current approaches in the pre-treatment of bone for radiocarbon dating by AMS, Radiocarbon, vol. 34, n 3, p. 279-291.

LANGLAIS M. (2007) - Dynamiques culturelles des sociétés magdaléniennes dans leurs cadres environnementaux. Enquête sur 7000 ans d'évolution de leurs équipements lithiques, thèse de doctorat, université de Toulouse II-Le Mirail et université de Barcelone, $550 \mathrm{p}$.

LONGIN R. (1971) - New method of collagen extraction for radiocarbon dating, Nature, vol. 230, p. 241-242.

NORMAND C. dir. (2008) - Grotte d'Isturitz. Opération de fouilles programmées tri-annuelles 2006-2008. Rapport final, Hasparren, rapport dactylographié.

PÉTILLON J.-M. (2004) - Lecture critique de la stratigraphie magdalénienne de la Grande Salle d'Isturitz (Pyrénées-Atlantiques), Antiquités nationales, t. 36, p. 105-131.

PÉTILLON J.-M. (2006) - Des Magdaléniens en armes. Technologie des armatures de projectile en bois de Cervidé du Magdalénien supérieur de la grotte d'Isturitz (Pyrénées-Atlantiques), Artefacts, 10, CEDARC, Treignes, $302 \mathrm{p}$. 
PÉTILLON J.-M. (2008) - First evidence of a whale-bone industry in the western European Upper Palaeolithic: Magdalenian artifacts from Isturitz (Pyrénées-Atlantiques, France), Journal of Human Evolution, vol. 54 (5), p. 720-726.

VAN KLINKEN G.J. (1999) - Bone collagen quality indicators for palaeodietary and radiocarbon measurements, Journal of Archaeological Science, vol. 26, p. 687-695.

Carolyn SZMIDT

Archaeology Centre, University of Toronto 19, Russell Street, Toronto, Ontario, M5S 2S2, Canada c.szmidt@utoronto.ca

Jean-Marc PÉTILLON

CNRS, TRACES, Université Toulouse II Maison de la recherche, 5, allées A. Machado

F-31058 Toulouse Cedex petillon@univ-tlse2.fr
Pierre CATTELAIN

ULB Treignes, écomusée du Viroin

asbl DIRE

81, rue de la Gare, 5670 Treignes, Belgique pcattela@ulb.ac.be

\section{Christian NORMAND}

SRA Aquitaine

Centre archéologique départemental 54, rue F. Jammes, F-64240 Hasparren cpjnormand@wanadoo.fr

Catherine SCHWAB

Musée d'Archéologie nationale Château, place Charles de Gaulle F-78105 Saint-Germain-en-Laye Cedex catherine.schwab@culture.gouv.fr 